Supporting information for

\title{
Synthesis of tertiary benzamides via Pd-catalyzed coupling of arylboronic esters and carbamoyl chlorides
}

\author{
Morten Lysén, Susan Kelleher, Mikael Begtrup, Jesper Langgaard Kristensen* \\ Department of Medicinal Chemistry, Danish University of Pharmaceutical Sciences, \\ Universitetsparken 2, DK-2100 Copenhagen, Denmark. \\ jekr@dfuni.dk
}

Table of content for supporting onformation

S1-S3

Experimental procedure and charaterisation of compounds

S4-S6

${ }^{1} \mathrm{H}$-spectra of compunds $\mathbf{3 d}, \mathbf{3 e}$ and $\mathbf{3 g}$

\section{General Procedure for the coupling of arylboronic esters and carbamoyl chlorides:}

Arylboronic ester (2.00 mmol), $\mathrm{CsF}(608 \mathrm{mg}, 4.00 \mathrm{mmol}), \mathrm{PdCl}_{2}\left(\mathrm{PPh}_{3}\right)_{2}$ (42.0 mg, $0.06 \mathrm{mmol}$ ) and carbamoyl chloride $(4.00 \mathrm{mmol})$ were dissolved in THF $(8 \mathrm{~mL})$ under $\mathrm{N}_{2}$. The mixture was heated at $80^{\circ} \mathrm{C}$ for $16 \mathrm{~h}$. At rt. the mixture was evaporated onto celite and purified by FC using EtOAc/heptane as eluent.

3a: 4-Benzoylmorpholine. ${ }^{1}$

The product was isolated as a colourless solid in $79 \%$ yield. Mp. $71-72{ }^{\circ} \mathrm{C}$. (reported $72{ }^{\circ} \mathrm{C}$ ). ${ }^{1} \mathrm{H}$ - and ${ }^{13} \mathrm{C}$ NMR data are in full agreement with reported values.

3b: 4-(2-Fluorobenzoyl)-morpholine. ${ }^{2}$

The product was obtained as an yellow solid in $87 \%$ yield. Mp. $75-77^{\circ} \mathrm{C}$. (reported $\left.77^{\circ} \mathrm{C}\right){ }^{1} \mathrm{H}-\mathrm{NMR}(300$ $\left.\mathrm{MHz}, \mathrm{CDCl}_{3}\right): \delta$ 7.41-7.36 (m, 2H), 7.22-7.18 (m, 1H), 7.11-7.04 (m, 1H), 3.81-3.75 (m, 4H), 3.65-3.62 (m, 2H), 3.38-3.34 (m, 2H). ${ }^{13} \mathrm{C}-\mathrm{NMR}\left(75 \mathrm{MHz}, \mathrm{CDCl}_{3}\right): \delta 164.6(\mathrm{~s}), 157.4\left(\mathrm{~s}, \mathrm{~d} J_{\mathrm{C}-\mathrm{F}}=246 \mathrm{~Hz}\right), 131.0\left(\mathrm{~d}, \mathrm{~d} J_{\mathrm{C}-\mathrm{F}}\right.$ $=8 \mathrm{~Hz}), 128.7\left(\mathrm{~d}, \mathrm{~d} J_{\mathrm{C}-\mathrm{F}}=4 \mathrm{~Hz}\right), 124.3\left(\mathrm{~d}, \mathrm{~d} J_{\mathrm{C}-\mathrm{F}}=3 \mathrm{~Hz}\right), 123.1\left(\mathrm{~s}, \mathrm{~d} J_{\mathrm{C}-\mathrm{F}}=17 \mathrm{~Hz}\right), 115.3\left(\mathrm{~d}, \mathrm{~d} J_{\mathrm{C}-\mathrm{F}}=21 \mathrm{~Hz}\right)$, $66.3(\mathrm{t}), 66.2(\mathrm{t}), 47.0(\mathrm{t}), 42.0(\mathrm{t})$.

3c: 4-(2-Chlorobenzoyl)-morpholine. ${ }^{3}$

The product was obtained as a white solid in $94 \%$ yield. $\mathrm{Mp} 72-73^{\circ} \mathrm{C}\left(\right.$ reported $73^{\circ} \mathrm{C}$ )

${ }^{1} \mathrm{H}-\mathrm{NMR}\left(300 \mathrm{MHz}, \mathrm{CDCl}_{3}\right)$ : $\delta$ 7.45-7.22 (m, 4H), 3.96-3.83 (m, 1H), 3.82-3.66 (m, 4H), 3.64-3.55 (m, $\left.1 \mathrm{H}\right)$, 3.35-3.14 (m, 2H). ${ }^{13} \mathrm{C}-\mathrm{NMR}$ (75 MHz, $\mathrm{CDCl}_{3}$ ): $\delta 165.9$ (s), 134.6 (s), 129.6 (d), 129.3 (s), 128.8 (d), 127.0 $(\mathrm{d}), 126.6(\mathrm{~d}), 66.0(\mathrm{t}), 65.9(\mathrm{t}), 46.4(\mathrm{t}), 41.3(\mathrm{t})$. 
3d: 4-(2-Cyanobenzoyl)-morpholine.

The product was obtained as an off-white solid in $87 \%$ yield. Mp. (EtOAc/PE) $107-109^{\circ} \mathrm{C}$.

${ }^{1} \mathrm{H}-\mathrm{NMR}\left(300 \mathrm{MHz}, \mathrm{CDCl}_{3}\right): \delta 7.73(\mathrm{~d}, 1 \mathrm{H}, J=7.7 \mathrm{~Hz}), 7.69(\mathrm{dt}, 1 \mathrm{H}, J=7.7,1.2 \mathrm{~Hz}), 7.55(\mathrm{dt}, 1 \mathrm{H}, J=7.7$, $1.2 \mathrm{~Hz}), 7.47(\mathrm{~d}, 1 \mathrm{H}, J=7.7 \mathrm{~Hz}), 3.91-3.78(\mathrm{mb}, 4 \mathrm{H}), 3.74-3.64(\mathrm{~m}, 2 \mathrm{H}), 3.37-3.26(\mathrm{~m}, 2 \mathrm{H}) .{ }^{13} \mathrm{C}-\mathrm{NMR}(75$ $\mathrm{MHz}, \mathrm{CDCl}_{3}$ ): $\delta 166.2$ (s), 139.4 (s), 133.1 (d), 132.9 (d), 129.7 (d), 127.5 (d), 116.7 (s), 109.9 (s), 66.6 (t), $47.6(\mathrm{t}), 42.5(\mathrm{t})$. Anal. Calc. for $\mathrm{C}_{12} \mathrm{H}_{12} \mathrm{~N}_{2} \mathrm{O}_{2}$ : C: 66.65, H: 5.59, N: 12.96; found: C: 66.74, H: 5.63, O: 12.83. Copy of ${ }^{1} \mathrm{H}-\mathrm{NMR}$ spectrum included, see page $\mathrm{S} 4$.

3e. 4-(2-Phenylbenzoyl)-morpholine.

The product was obtained as a white solid in $76 \%$ yield. Mp. (EtOAc/PE) $127-128^{\circ} \mathrm{C}$.

${ }^{1} \mathrm{H}-\mathrm{NMR}\left(300 \mathrm{MHz}, \mathrm{CDCl}_{3}\right): \delta$ 7.50-7.46 (m, 9H), $3.70(\mathrm{~m}, 1 \mathrm{H}), 3.59(\mathrm{ddd}, 1 \mathrm{H}, J=11.2,5.0,3.0 \mathrm{~Hz}), 3.43$ (ddd, 1H, $J=13.4,7.9,3.2 \mathrm{~Hz}), 3.27$ (m, 2H), 2.95 (ddd, 1H, $J=13.4,7.9,3.1 \mathrm{~Hz}), 2.72$ (ddd, 1H, $J=13.4$, 4.9, $4.6 \mathrm{~Hz}) 2.43(\mathrm{ddd}, 1 \mathrm{H}, J=11,1,7.9,2.9 \mathrm{~Hz}) .{ }^{13} \mathrm{C}-\mathrm{NMR}\left(75 \mathrm{MHz}, \mathrm{CDCl}_{3}\right): \delta 169.6$ (s), $139.4(\mathrm{~s}), 138.3$ (s), 134.5 (s), 129.4 (d), 129.0 (d), 128.6 (d), 128.4 (d), 127.8 (d), 127.6 (d), 66.0 (t), 65.9 (t), $46.7(\mathrm{t}), 41.7$ (t). Anal. Calc. for $\mathrm{C}_{17} \mathrm{H}_{17} \mathrm{NO}_{2}$ : C: 76.38, H: 6.41, N: 5.24; found: C: 76.11, H: 6.14, O: 5.11. Copy of ${ }^{1} \mathrm{H}-$ NMR spectrum included, see page S5.

3f: 4-(2-Methoxybenzoyl)-morpholine. ${ }^{4}$

The product was obtained as a white solid in $86 \%$ yield. Mp. $52-53{ }^{\circ} \mathrm{C}$. (reported $50-52^{\circ} \mathrm{C}$ ) ${ }^{1} \mathrm{H}$ - and ${ }^{13} \mathrm{C}-\mathrm{NMR}$ data are in full agreement with reported values.

3g. tert-Butyl 2-(morpholine-4-carbonyl)phenylcarbamate.

The product was obtained as an yellow solid in $79 \%$ yield. Mp. (EtOAc/PE) $136-138^{\circ} \mathrm{C}$.

${ }^{1} \mathrm{H}-\mathrm{NMR}\left(300 \mathrm{MHz}, \mathrm{CDCl}_{3}\right): \delta 8.09(\mathrm{~d}, 1 \mathrm{H}, J=8.3 \mathrm{~Hz})$ ), $7.86(\mathrm{sb}, 1 \mathrm{H}), 7.37$ (ddd, $\left.1 \mathrm{H}, J=8.6,7.4,1.6 \mathrm{~Hz}\right)$, $7.15(\mathrm{dd}, 1 \mathrm{H}, J=7.6,1.5 \mathrm{~Hz}), 7.02(\mathrm{dt}, 1 \mathrm{H}, J=7.5,1.0 \mathrm{~Hz}), 3.86-3.57(\mathrm{sb}, 8 \mathrm{H}), 1.51(\mathrm{~s}, 9 \mathrm{H}) .{ }^{13} \mathrm{C}-\mathrm{NMR}(75$ $\mathrm{MHz}, \mathrm{CDCl}_{3}$ ): $\delta 168.7$ (s), 152.6 (s), 137.2 (s), 130.6 (d), 127.1 (d), 122.8 (s), 121.8 (d), 121.0 (d), 80.4 (t), 66.7 (t), 28.2 (q). Anal. Calc. for $\mathrm{C}_{16} \mathrm{H}_{22} \mathrm{~N}_{2} \mathrm{O}_{4}$ : C: 62.73, H: 7.24, N: 9.14; found: C: 62.71, H: 7.04, O: 8.99. Copy of ${ }^{1} \mathrm{H}-\mathrm{NMR}$ spectrum included, see page $\mathrm{S} 6$.

3h: 2-Cyano- $N, N$-dimethylbenzamide. ${ }^{5}$

The product was obtained as a colourless oil in $93 \%$ yield. ${ }^{1} \mathrm{H}$ - and ${ }^{13} \mathrm{C}-\mathrm{NMR}$ data are in full agreement with reported values. 
3i: $N, N$-Diethylbenzamide. ${ }^{6}$

The product was obtained as a colourless oil in $85 \%$ yield. ${ }^{1} \mathrm{H}$ - and ${ }^{13} \mathrm{C}-\mathrm{NMR}$ data are in full agreement with reported values.

3j: $N, N$-Diethyl-2-methoxybenzamide. ${ }^{7}$

The product was obtained as a colourless oil in $74 \%$ yield. ${ }^{1} \mathrm{H}$ - and ${ }^{13} \mathrm{C}-\mathrm{NMR}$ data are in full agreement with reported values.

3k: Ethyl 2-(diethylcarbamoyl)benzoate. ${ }^{8}$

The product was obtained as a colourless oil in $51 \%$ yield. ${ }^{1} \mathrm{H}$ - and ${ }^{13} \mathrm{C}-\mathrm{NMR}$ data are in full agreement with reported values.

31: (2-Fluorobenzoyl)-4-methylpiperazine. ${ }^{9}$

The product was obtained as an yellow oil in $74 \%$ yield. ${ }^{1} \mathrm{H}-\mathrm{NMR}\left(300 \mathrm{MHz}, \mathrm{CDCl}_{3}\right): \delta 7.41-7.32(\mathrm{~m}, 2 \mathrm{H})$, 7.21-7.14 (m, 1H), 7.10-7.02 (m, 1H), 3.84-3.80 (m, 2H), 3.34-3.30 (m, 2H), $2.48(\mathrm{t}, 2 \mathrm{H}, J=5.7 \mathrm{~Hz}), 2.37-$ $2.33(\mathrm{~m}, 2 \mathrm{H}), 2.32(\mathrm{~s}, 3 \mathrm{H}) .{ }^{13} \mathrm{C}-\mathrm{NMR}\left(75 \mathrm{MHz}, \mathrm{CDCl}_{3}\right): \delta 163.7(\mathrm{~s}), 156.8\left(\mathrm{~s}, \mathrm{~d} J_{\mathrm{C}-\mathrm{F}}=247 \mathrm{~Hz}\right), 130.2(\mathrm{~d}, \mathrm{~d}$ $\left.J_{\mathrm{C}-\mathrm{F}}=8 \mathrm{~Hz}\right), 127.9\left(\mathrm{~d}, \mathrm{~d} J_{\mathrm{C}-\mathrm{F}}=4 \mathrm{~Hz}\right), 123.6\left(\mathrm{~d}, \mathrm{~d} J_{\mathrm{C}-\mathrm{F}}=3 \mathrm{~Hz}\right), 123.0\left(\mathrm{~s}, \mathrm{~d} J_{\mathrm{C}-\mathrm{F}}=18 \mathrm{~Hz}\right), 114.6\left(\mathrm{~d}, \mathrm{~d} J_{\mathrm{C}-\mathrm{F}}=21\right.$ $\mathrm{Hz}), 54.0(\mathrm{t}), 53.5(\mathrm{t}), 45.9(\mathrm{t}), 44.9(\mathrm{q}), 40.7(\mathrm{t})$.

\section{References}

1) Tillack, A.; Rudloff, I.; Beller, M. Eur. J. Org. Chem. 2001, 3, 523

2) Fukui et al.; Chem. Abstr.; 1960; 5518.

3) Kuliev, A. B.; Kurbanov, M. M.; Mamedov, A. G.; Kuliev, F. A.; J. Appl. Chem. USSR (Engl. Transl.). 1985, 58, 139.

4) Woodgate, P.D.; Sutherland, H.S.; Rickard, C.E.F. J. Organomet. Chem. 2001; 626, 199.

5) Borch,R.F. Grudzinskas, C.V.; Peterson, D.A.; Weber, L.D. J. Org. Chem. 1972, 37, 1141.

6) Hans, J.J.; Driver, R.W.; Burke, S.D. J. Org. Chem. 2000, 65, 2114.

7) Ardus, J.A.; Gillman, I.G.; Manderville, R.A. Can. J. Chem. 1998, 76, 907.

8) Raber, D.J.; Gariano, P.; Brod, A.O.; Gariano, A.; Guida, W.C.; Guida, A.R.; Herbs, M.D. J. Org. Chem. 1979, 44,1149

9) Johnson,H.L. Skinner, W.; Skidmore, D.; Maibach, H. J. Med. Chem. 1968, 11, 1265. 


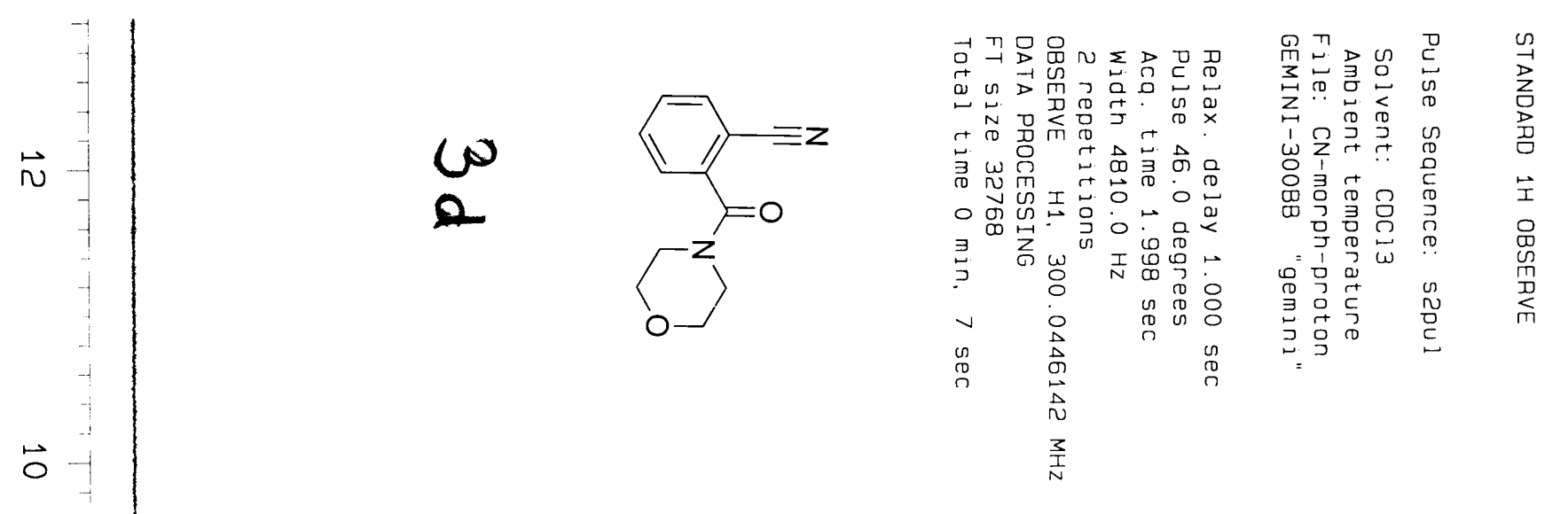

$\infty$

$\sigma$

$\triangle$

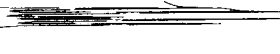

$N$
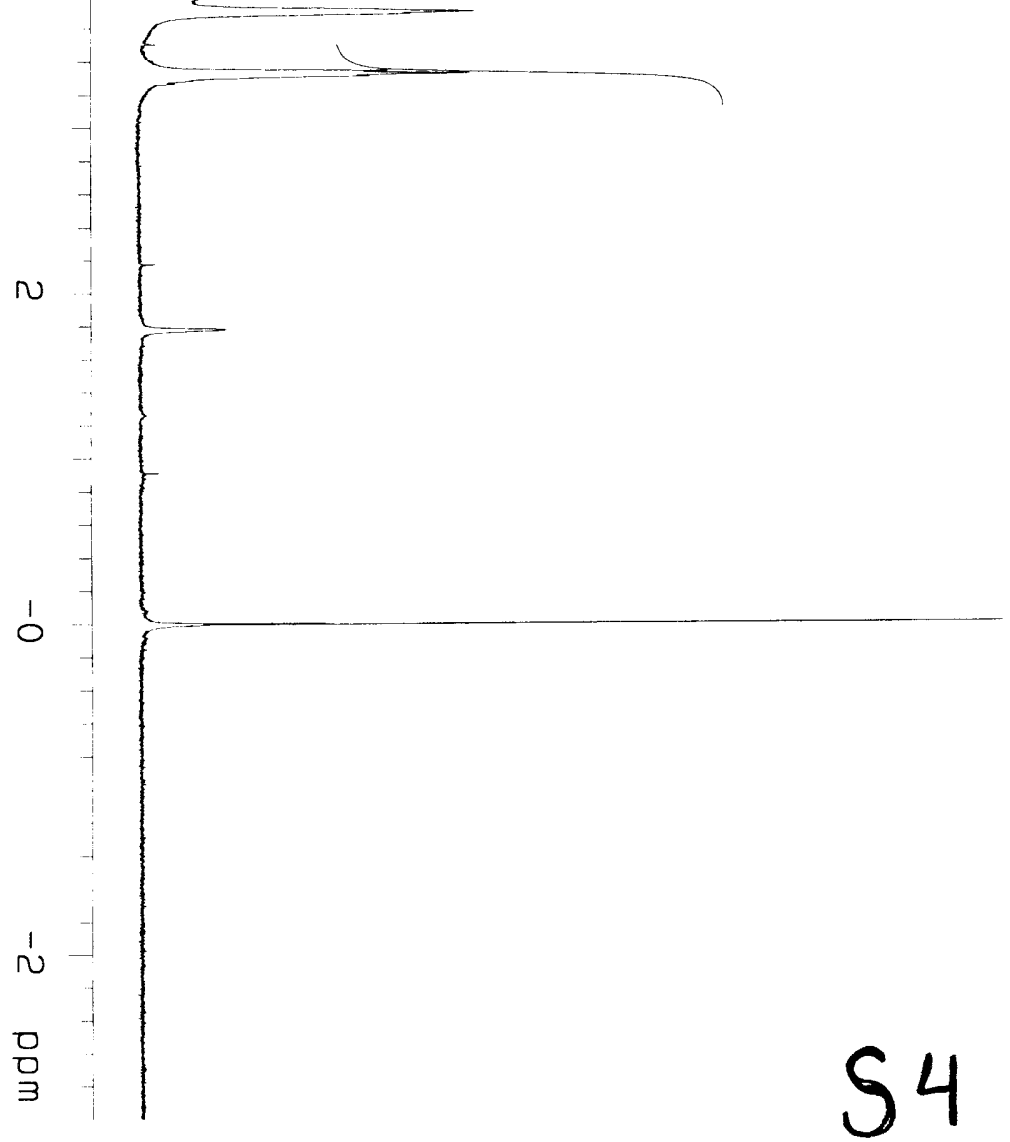


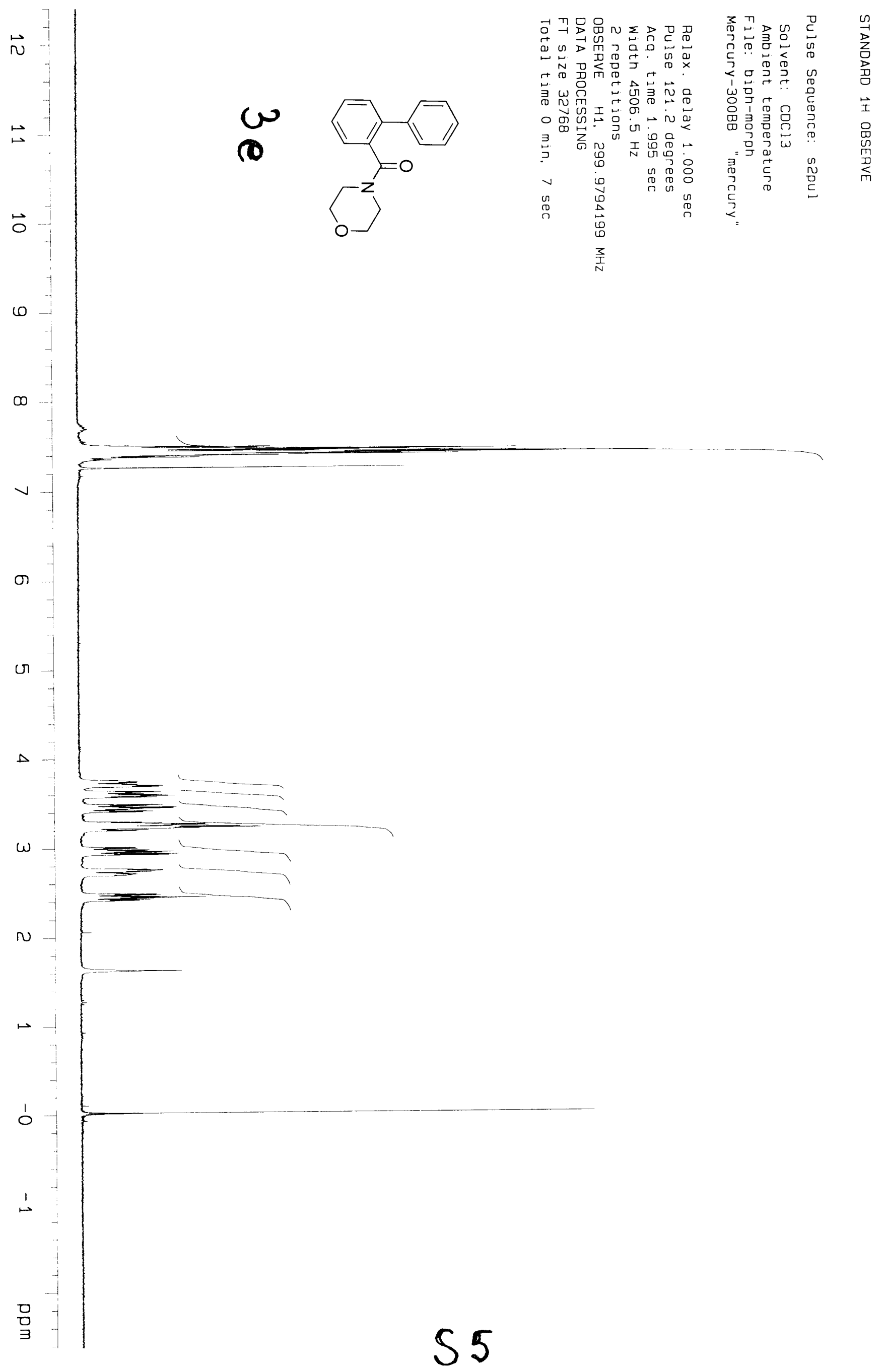




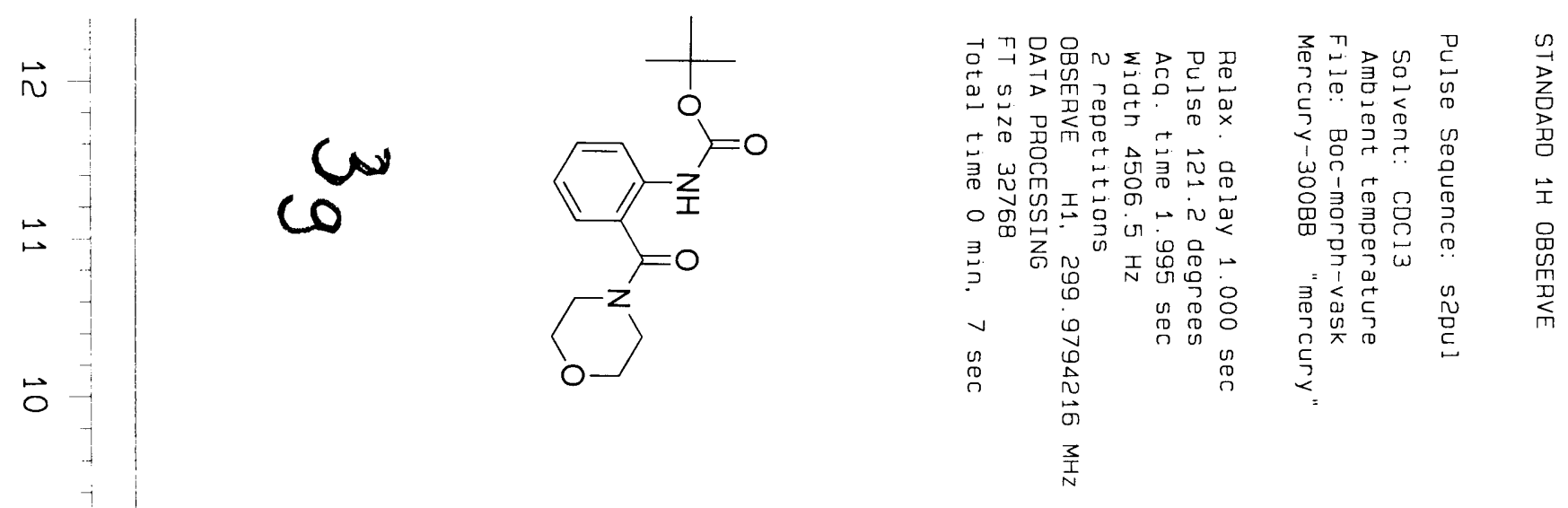

$\omega$

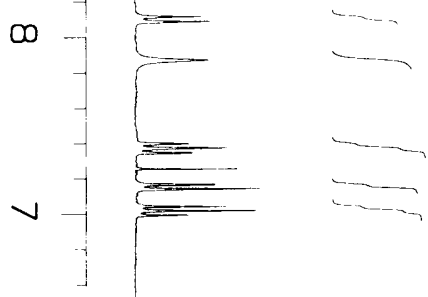

б

$\triangle$

$G$

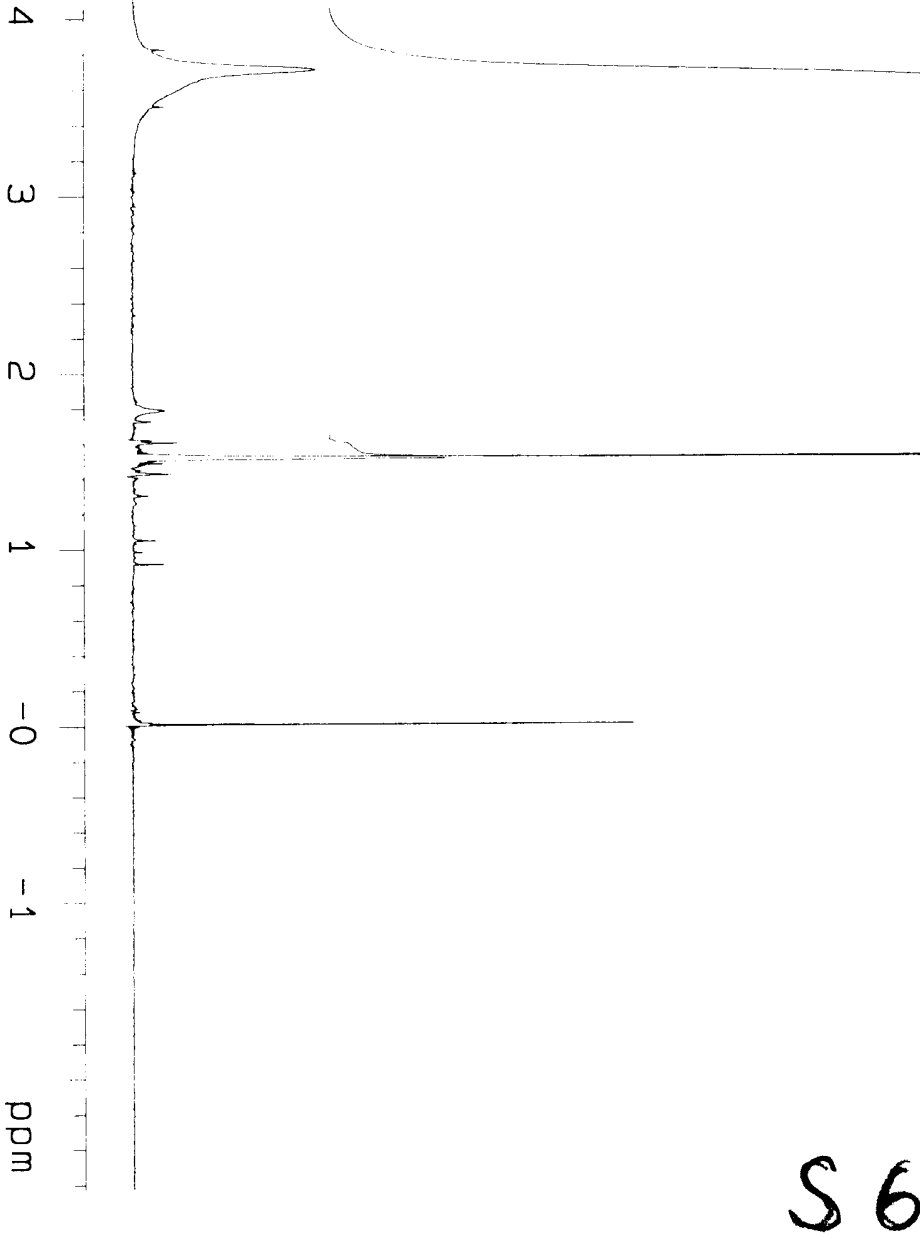

